\title{
Detection of Exomoons Inside the Habitable Zone
}

\author{
Luis Ricardo M. Tusnski ${ }^{1}$ and Adriana Valio ${ }^{2}$ \\ ${ }^{1}$ INPE, Sao Jose dos Campos, Brazil \\ lrtusnski@gmail.com \\ ${ }^{2}$ CRAAM, Mackenzie University, Sao Paulo, Brazil \\ adrivalio@gmail.com
}

\begin{abstract}
Since the discovery of the first exoplanets, those most adequate for life to begin and evolve have been sought. Due to observational bias, however, most of the discovered planets so far are gas giants, precluding their habitability. However, if these hot Jupiters are located in the habitable zones of their host stars, and if rocky moons orbit them, then these moons may be habitable. In this work, we present a model for planetary transit simulation considering the presence of moons around a planet. The moon orbit is considered to be circular and coplanar with the planetary orbit. The other physical and orbital parameters of the star, planet, and moon, can be adjusted in each simulation. It is possible to simulate as many successive transits as desired. Since the presence of spots on the surface of the star may produce a signal similar to that of the presence of a moon, our model also allows for the inclusion of starspots. The goal is to determine the criteria for detectability of moons using photometry with the CoRoT and Kepler telescopes taking into account the stellar activity.
\end{abstract}

Keywords. planets and satellites: general, techniques: photometric

\section{Introduction}

At the time of the writing of this article, more than 990 exoplanets were known (www.exoplanet.eu). Most of these are Hot Jupiters, gas-giants very close to their parent stars. In the last few years, the possibility of these planets having moons has been studied. One of the reasons for this supposition is that all gas-giant planets in the Solar System have moons. If a gas-giant planet is inside the habitable zone (the region around the star where an Earth-like planet with adequate atmospheric conditions can hold water in liquid state on its surface) of the star, then its exomoons may be habitable.

Several methods have been proposed for exomoons detection, but so far no exomoon has been detected. In a previous work (Tusnski \& Valio, 2011), we proposed a model to simulate the planet+moon transit in front of the host star. We showed that these transit events cause small deformations in the light curve, in the form of steps (before the ingress or after the egress) or assymetries (in the bottom part of the curve). This technique was first suggested by Sartoretti \& Schneider (1999). We described how these ligthcurve signatures can be used to detect exomoons, and stablished that CoRoT can detect exomoons down to $1.3 R_{\oplus}$ in radius. For Kepler, this detection limit is $0.3 R_{\oplus}$. In both cases, we considered a Jupiter-like planet with 45-day period. Our model is similar to light curve simulations of multi-body occultations of stars, planets, rings and moons that have been implemented in the program UTM by Deeg (2009), using pixelized representations of these bodies. The main difference is that our model permits the inclusion of spots on the surface of the star. Another similar model was suggested by Kipping (2011), with the difference that his model is analytical, in contrast with our pixelization model. 


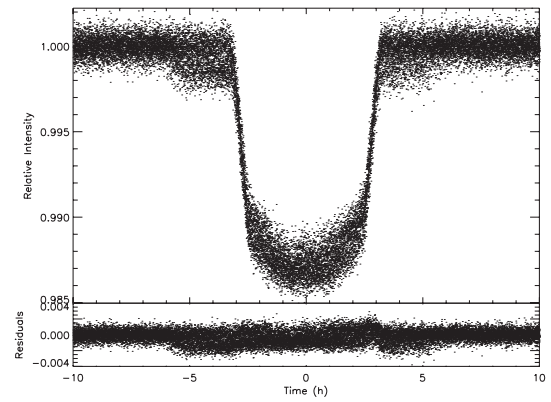

(a)

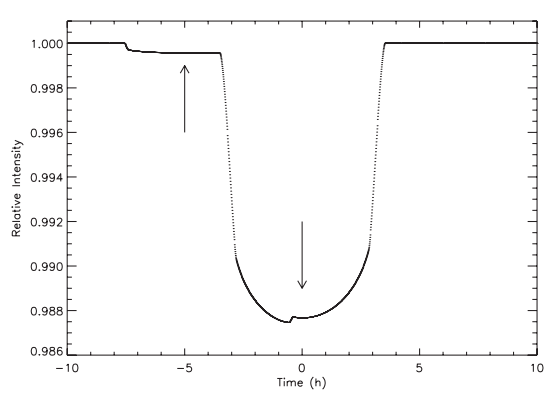

(b)

Figure 1. (a) Example of the residual method for exomoons detection, done with our simulaiton model. Superposition of 50 transits of a 45-day period Jupiter-like planet. (b) Photometric distortions caused by an Earth-like exomoon around a 45-day period Jupiter-like planet.

In the present work, we are using our simulation model together with other detection techniques to actually search for exomoons. Our goal is to look for moons around habitable planets because of the exobiological interest in these objects. However, the detection of exomoons around non-habitable planets is also of great interest for reasons such as planet formation models, dynamic stability studies, models of moon interior, moon-planet interactions, among others.

\section{Development}

To search for exomoons, we have built an IDL program that receives as input the FITS files containing the light curves. We can use Kepler or CoRoT light curves. The program uses 3 different methods to look for an indication of the exomoon. If any signal is detected, we can confirm the detection and measure the orbital parameters using our model.

\subsection{The Residuals Method}

This is a very simple indication on the presence of a moon around an exoplanet suggested by Szabo et al. (2011). They noted that when we have a great number of transits, we can superpose them all; if the planet hosts an exomoon, an excess in the residuals of the light curve is seen. This residual excess is caused by the amplified effect of photometric signatures of the moon. Figure 2.1 shows an example of this technique.

\subsection{Photometric Distortions}

The exomoon causes some distortions in the transit light curve as it passes in front of the planet-hosting star. These signatures appear differently in each transit, due to the exomoon position relative to the exoplanet. There are two main types of signatures: steps appearing before the ingress or after the egress region, or assymetries appearing mainly in the bottom part of the light curve. The steps occur when the moon enters or leaves the transit regions separate from the planet, and the assymetries are caused by mutual events including planet and moon (eclipses).

Similar to planetary transits, the step depth is related to the exomoon radius and its periodicity indicates the orbital period of the moon around the planet. In our study, we considered a case where the exomoon orbital plane is coplanar with the planetary orbital plane. Figure 2.1 shows this distortions in a simulated light curve. 


\subsection{Timing Variations}

Besides the photometric distortions, Sartoretti \& Schneider (1999), Kipping (2009a), Kipping (2009b) and Haghighipour et al. (2013) noted two other effects caused by the presence of an exomoon around an exoplanet: the variation on the transit central time (Transit Time Variation, TTV) and on the transit duration (Transit Duration Variation, TDV). These variations are caused by the planet position and movement around the planet-moon barycenter. Together, these two effects lead to the measurement of the moon orbital period and mass.

\section{The Search for Exomoons}

As mentioned before, our program receives the input light curves as FITS files, and performs a series of calculations using the discussed methods. Using BLS algorithm (Kovacs et al. 2002), the planet orbital period is calculated. This value is compared to the literature for verification. After that, the program separates successive transits and measures its central and duration times. All transits are plotted in PS files for future analysis and search for photometric distortions by visual inspection. Finally, the transits are overlayed for residual analysis.

The goal of the program is to identify candidates for planets hosting exomoons. After this identification, other measurements using our transit model are performed to obtain the exomoon's parameters. Our objetive is to apply this program to CoRoT and Kepler light curves.

\section{Preliminary Results and Analysis}

This program has been applied to some Kepler and CoRoT single-planet light curves. This simplification ensures that timing effects are not caused by additional planets in the system. For the first analysis, we selected CoRoT-2b, CoRoT-3b, CoRoT-4b, CoRoT-5b, CoRoT-6b, CoRoT-8b, CoRoT-9b and all the Kepler confirmed single-planets. So far, no signal of the presence of a moon was detected within the detection limits.

The future steps of our search include a review in the transit model, removing some simple assumptions made and analysis of the Kepler planetary candidates, begining with the most likely to host a habitable exomoon.

\section{Acknowledgements}

The authors would like to thank the Brazilian agency FAPESP (grants 2006/50654-3 and 2010/17814-2 ) for the financial support of this research.

\section{References}

Deeg, H. J. 2009, IAU Symp. 253, 388

Haghighipour, N., Capen, S., \& Hinse, T. C. 2013, CeMDA, 117, 75

Kipping, D. M. 2009, MNRAS, 392, 181

Kipping, D. M. 2009, MNRAS, 396, 1797

Kipping, D. M. 2011, MNRAS, 416, 689

Kovacs, G., Zucker, S., \& Mazeh, T. 2002, A\&AA, 391, p. 369

Sartoretti, P. \& Schneider, J. 1999, A\&AS, 134, 553

Szabo, G. M., Simon, A. E., Kiss, L., \& Regaly 2011, IAU Symp. 276, 556

Tusnski, L. R. M .\& Valio, A. 2011, ApJ 743, 97 